\title{
Hydrogen migration in single crystal and polycrystalline zinc oxide
}

\author{
N. H. Nickel \\ Hahn-Meitner-Institut Berlin, Kekuléstrasse 5, 12489 Berlin, Germany \\ (Received 23 December 2005; revised manuscript received 13 March 2006; published 12 May 2006)
}

\begin{abstract}
Hydrogen diffusion in single crystal and polycrystalline zinc oxide was investigated by deuterium diffusion and hydrogen effusion experiments. Deuterium concentration depth profiles were measured as a function of the passivation temperature, while in $\mathrm{H}$ effusion experiments the molecular hydrogen flux was measured as a function of the heating rate. The diffusion coefficient exhibits thermally activated behavior and varies between $E_{A}=0.17$ and $0.37 \mathrm{eV}$. The change of $E_{A}$ is accompanied by a change of the diffusion prefactor by eight orders of magnitude. This indicates that $E_{A}$ is not related to the energetic position of $\mathrm{H}$ transport sites or the barrier height between such sites. Using the microscopic diffusion prefactor, the position of the hydrogen chemical potential, $\mu_{H}$, was estimated. With increasing temperature, $\mu_{H}$ decreases with a rate of $\approx 0.0013 \mathrm{eV} / \mathrm{K}$. At $\mathrm{H}$ concentrations of less than $10^{17} \mathrm{~cm}^{-3} \mu_{H}$ is pinned. The hydrogen density of states was derived from $\mathrm{H}$ effusion data, which is consistent with a diffusion activation of about $1.0 \mathrm{eV}$ as was originally reported by Mollwo [Z. Phys. 138, 478 (1954)] and Thomas and Lander [J. Chem. Phys. 25, 1136 (1956)]. Clear evidence for hydrogen deep traps was found in single crystal and polycrystalline $\mathrm{ZnO}$.
\end{abstract}

DOI: 10.1103/PhysRevB.73.195204

PACS number(s): 66.30.-h, 81.05.Dz

\section{INTRODUCTION}

Recently, zinc oxide $(\mathrm{ZnO})$ has regained interest for a number of reasons. Because of its large energy gap of $3.4 \mathrm{eV}, \mathrm{ZnO}$ has been utilized as active layer in transparent thin-film transistors. ${ }^{1,2}$ On the other hand, because of the large exciton binding energy of about $60 \mathrm{meV},{ }^{3} \mathrm{ZnO}$ is considered as a superior material for light-emitting diodes and semiconductor lasers. However, prior to the investigation and fabrication of optoelectronic devices, a major obstacle has to be overcome; namely, to achieve $p$-type conductivity with sufficiently high hole concentrations.

Besides intrinsic defects in $\mathrm{ZnO}$, such as $\mathrm{Zn}$ interstitials that cause $n$-type conductivity, hydrogen has been identified as a shallow donor. According to calculations based on density-functional theory, the $\mathrm{H}$ atom forms a strong bond with an oxygen atom causing a large displacement of the adjacent $\mathrm{Zn}$ atom. This complex was suggested as an $\mathrm{H}$ donor complex. ${ }^{4}$ Muon spin rotation experiments ${ }^{5}$ and nuclear double resonance experiments ${ }^{6}$ confirmed the theoretical prediction. Hydrogen is readily incorporated into $\mathrm{ZnO}$ during processing and/or post-treatment steps. Since it turned out that state-of-the-art single crystal $\mathrm{ZnO}$ samples contain up to $10^{17} \mathrm{~cm}^{-3} \mathrm{H}$ atoms, ${ }^{7,8}$ which can impede effective $p$-type doping, it became apparent that a fundamental understanding of hydrogen accommodation and migration is of great importance.

The role of hydrogen in $\mathrm{ZnO}$ has already been investigated in great detail in the 1950 s. Heating $\mathrm{ZnO}$ crystals in a hydrogen atmosphere resulted in an increase of the conductivity, and when the hydrogen was subsequently removed the conductivity returned to its original value. ${ }^{9}$ Assuming that all hydrogen atoms ionize and that the mobility of the electrons is independent of concentration, information on hydrogen diffusion and solubility was obtained from conductivity measurements. ${ }^{9,10}$ Mollwo 9 reported a diffusion activation energy of $1.12 \mathrm{eV}$. On the other hand, Thomas and Lander ${ }^{10}$ obtained a somewhat smaller value of $0.91 \mathrm{eV}$ because they argued that under the experimental conditions used for the measurements the electrons are more mobile than the protons and diffuse ahead dragging the protons along. This important topic was revisited recently. Hydrogen diffusion was investigated by exposing $\mathrm{ZnO}$ samples to a deuterium plasma. Subsequently, the D depth profiles were measured by secondary-ion-mass spectrometry (SIMS). Interestingly, the diffusion activation energy determined from deuterium concentration depth profiles was found to be as low as $0.17 \pm 0.12 \mathrm{eV}^{11}$

In this work, we investigate hydrogen transport in single crystal and polycrystalline $\mathrm{ZnO}$ by analyzing deuterium depth profiles obtained from SIMS and by analyzing hydrogen effusion spectra. We find that the diffusion activation energy, $E_{A}$, depends significantly on the experimental conditions. A change of $E_{A}$ from 0.17 to $0.37 \mathrm{eV}$ is accompanied by a change of the diffusion prefactor by about eight orders of magnitude. The implications of this observation on hydrogen transport are discussed in detail.

The paper is organized as follows. Section II describes the sample preparation and gives information on the experimental procedures used. Deuterium concentration profiles obtained on single crystal and polycrystalline $\mathrm{ZnO}$ are shown in Sec. III A. while the results from hydrogen effusion measurements are shown in Sec. III B. Implications of the data for $\mathrm{H}$ diffusion are discussed in Sec. IV, and finally Sec. V summarizes the main results of this research.

\section{EXPERIMENT}

The experiments described in this paper were performed on single crystal ${ }^{12}$ and polycrystalline $\mathrm{ZnO}$. The polycrystalline samples were grown on quartz substrates by dc magnetron sputtering at a substrate temperature of $523 \mathrm{~K}$. The sputtered $\mathrm{ZnO}$ samples had a thickness of $8 \mu \mathrm{m}$ and were composed of columnar grains. The length of the grains is 
limited only by the film thickness. All investigated samples were nominally undoped with a (0001) crystallographic orientation.

As a tracer element monatomic ${ }^{2} \mathrm{H}$ (deuterium) was introduced into the specimens using an optically isolated remote plasma. ${ }^{13}$ Deuterium (D) is a readily identifiable isotope, which duplicates hydrogen chemistry. During postdeuteration the concentration of monatomic $\mathrm{D}$ in the plasma is constant, and was estimated to be $\approx 5 \times 10^{15} \mathrm{~cm}^{-3}$ using electron spin resonance measurements. ${ }^{13}$ During plasma passivation, the sample temperature was kept constant and the hydrogenation time was typically $1 \mathrm{~h}$. At the end of the plasma passivation, the samples were rapidly cooled to room temperature with a cooling rate of about $20 \mathrm{~K} / \mathrm{s}$. Then, the specimens were taken out of the plasma passivation setup. After mounting the samples in a SIMS apparatus, the D depth distributions were measured using a $\mathrm{Cs}^{+}$-ion beam. An implanted $\mathrm{ZnO}$ standard was used to calibrate the deuterium concentrations. The depth scales were obtained by measuring the depth of the sputtered craters using a mechanical stylus.

For one set of single crystal, $\mathrm{ZnO}$ information on hydrogen migration was obtained from hydrogen effusion measurements. For this purpose, samples from a single crystal were each heated with a different heating rate, while the molecular hydrogen flux was measured with a quadrupole mass spectrometer. Prior to each measurement, background spectra were taken and subtracted from the sample data. The relative $\mathrm{H}_{2}$ flux of the measurements was calibrated using the known neon flux through a capillary. The analysis of the effusion data is described in more detail in Sec. III B.

In the subsequent discussion, the term hydrogenation will be used interchangeably with the term deuteration since no significant differences in its migration properties have been found for the two isotopes. ${ }^{14}$

\section{RESULTS}

The results presented in this section were obtained by varying the following experimental parameters: The type of $\mathrm{ZnO}$, e.g., single crystal and sputter deposited polycrystalline $\mathrm{ZnO}$, postdeuteration temperature, and heating rate in hydrogen effusion experiments. In Sec. III A, the results obtained from deuterium plasma diffusion experiments on single crystal and polycrystalline $\mathrm{ZnO}$ are shown. Section III B focuses on the results obtained from hydrogen effusion experiments performed on single crystal $\mathrm{ZnO}$.

\section{A. Diffusion from a plasma}

\section{Single crystal zinc oxide}

Figure 1 shows deuterium depth profiles obtained on single crystal $\mathrm{ZnO}$ after an exposure to monatomic D for $1 \mathrm{~h}$ at the indicated temperatures. For clarity, only two D depth profiles are shown. With increasing passivation temperature from 413 to $513 \mathrm{~K}$, the deuterium surface concentration, $\mathrm{C}_{0}$, increases from about $4 \times 10^{19}$ to $10^{20} \mathrm{~cm}^{-3}$. The $\mathrm{D}$ depth profiles can be divided into two regions. In the near-surface region (depth $<1.0 \mu \mathrm{m}$ ), the $\mathrm{D}$ concentration is enhanced and the deuterium profiles exhibit an exponential decay with

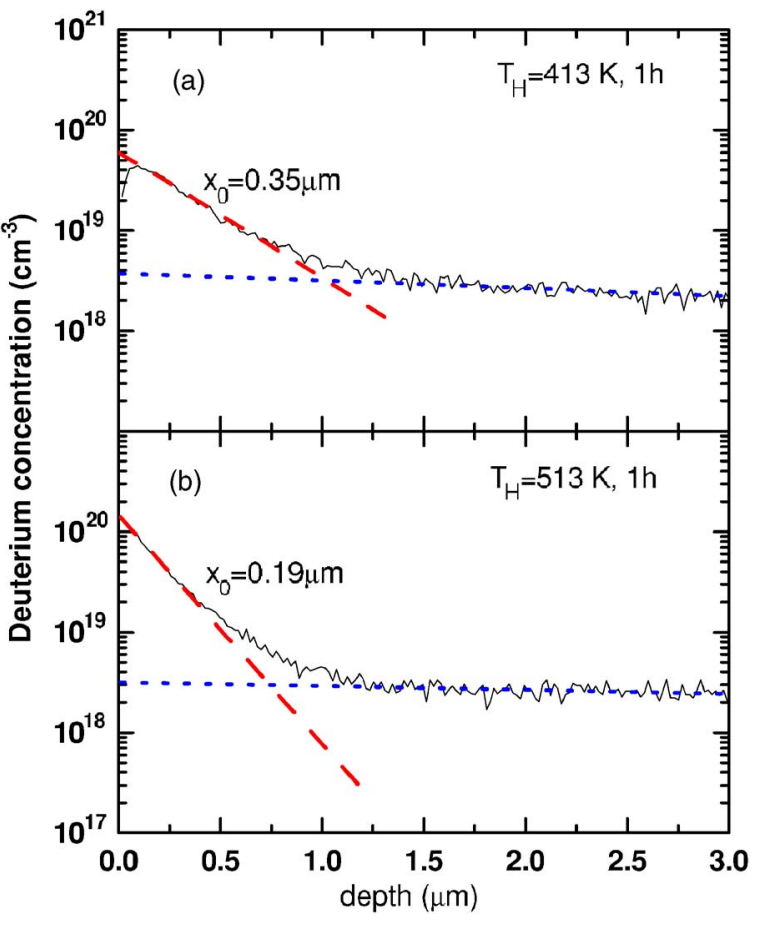

FIG. 1. (Color online) Deuterium concentration depth profiles in single crystal $\mathrm{ZnO}$ obtained from SIMS measurements. The samples were exposed to monatomic deuterium for $60 \mathrm{~min}$ at the indicated temperatures. The dashed and dotted lines represent least-squares fits to an exponential decay and an erfc, respectively. For clarity, only two D profiles are shown.

depth. At deuteration temperatures from 413 to $513 \mathrm{~K}$, a characteristic decay length, $x_{0}$, of 0.35 and $0.19 \mu \mathrm{m}$ was obtained, respectively. In this region, hydrogen diffusion is much slower than in the bulk of the samples. The diffusion coefficients were estimated using the relation

$$
D=\frac{x_{0}^{2}}{4 t_{D}}
$$

where $t_{D}$ is the deuteration time. With increasing temperature, the diffusion coefficient in the high $\mathrm{D}$ concentration region decreases from $\approx 3.3 \times 10^{-13}$ to $1 \times 10^{-14} \mathrm{~cm}^{2} / \mathrm{s}$. The dependence of the $\mathrm{D}$ depth profiles and the diffusion coefficient on the passivation temperature indicate that the concentration of near-surface traps decreases, but the remaining traps are deeper in energy retarding trapped $\mathrm{H}$ significantly. This will be discussed in more detail in Sec. IV B.

At a depth greater than $1.0 \mu \mathrm{m}$, the deuterium concentration is about one order of magnitude lower than in the nearsurface region, and the diffusion is enhanced. The diffusion coefficient was obtained from least-squares fits of the data to a complementary error function

$$
C_{D}(x, t)=C_{0} \operatorname{erfc}\left(\frac{x}{2 \sqrt{D t_{D}}}\right),
$$

where $C_{0}$ is the D surface concentration and $x$ is the depth. The fits are indicated by the dotted lines in Fig. 1 and are in good agreement with the data for a depth greater than $1 \mu \mathrm{m}$. 


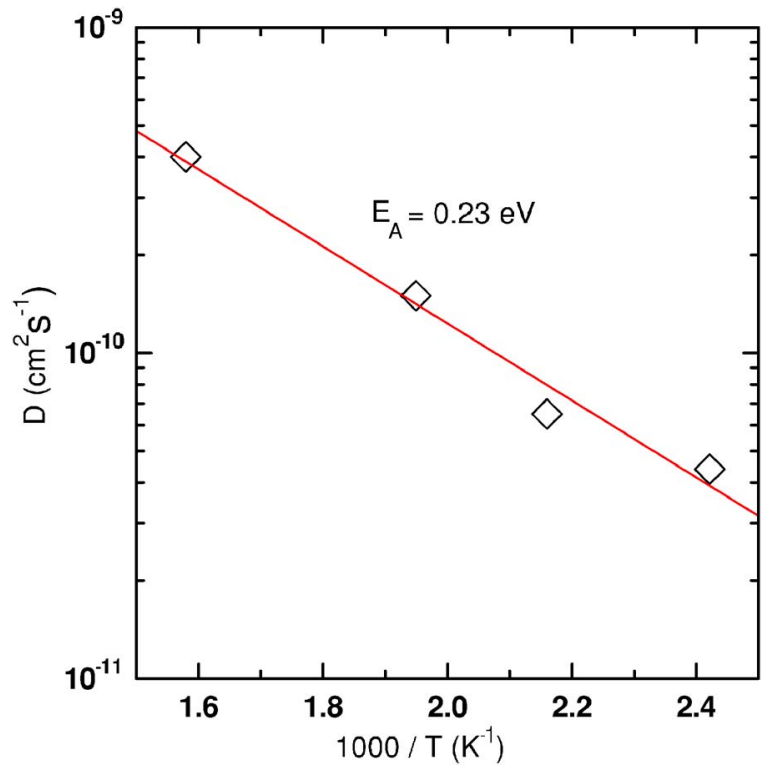

FIG. 2. (Color online) Diffusion coefficient as a function of the reciprocal temperature for deuterium diffusion from a plasma into single crystal $\mathrm{ZnO}$. The data are represented by the diamonds. The line is a least-squares fit to the data and exhibits an activated behavior with a slope of $E_{A}=0.23 \mathrm{eV}$.

This indicates that the fast diffusion component of the deuterium profiles can be described by a single diffusion coefficient.

The diffusion coefficients obtained from the least-squares fits are plotted in Fig. 2 versus $1 / T$. $D$ exhibits an apparent activation energy of $E_{A}=0.23 \mathrm{eV}$. Recently, a similar small value of $0.17 \pm 0.12 \mathrm{eV}$ was reported for hydrogen diffusion in bulk $\mathrm{ZnO}$, and it was concluded that such a low value is consistent with the idea that atomic hydrogen migrates via interstitial sites. ${ }^{11}$ However, both values for the activation energy are significantly smaller than previously reported values of $E_{A}=1.12$ and $0.91 \mathrm{eV}$ by Mollwo ${ }^{9}$ and Thomas and Lander, ${ }^{10}$ respectively.

\section{Polycrystalline $\mathrm{ZnO}$}

In order to investigate the influence of disorder similar deuterium diffusion experiments were performed using sputter deposited polycrystalline $\mathrm{ZnO}$ films. In Fig. 3, the D concentration as a function of the sample depth is shown. Compared to the data obtained for $\mathrm{D}$ diffusion in single crystal $\mathrm{ZnO}$ (Fig. 1) in polycrystalline $\mathrm{ZnO}$, a significant $\mathrm{D}$ concentration is accommodated within the first 2 to $3 \mu \mathrm{m}$. The D surface concentration reaches a constant value of 2-3 $\times 10^{20} \mathrm{~cm}^{-3}$. Then the $\mathrm{D}$ concentration decreases exponentially with a characteristic decay length from $x_{0} \approx 0.26$ to $0.37 \mu \mathrm{m}$. These values are comparable to $x_{0}$ determined from single crystal $\mathrm{ZnO}$. However, in contrast to single crystal $\mathrm{ZnO}$ in polycrystalline samples, the characteristic decay length and the amount of D trapped in the near-surface region increase with increasing passivation temperature.

At a depth greater than about $2 \mu \mathrm{m}$, the depth profiles deviate from an exponential decay and follow a complementary error function. According to the data shown in Fig. 3,

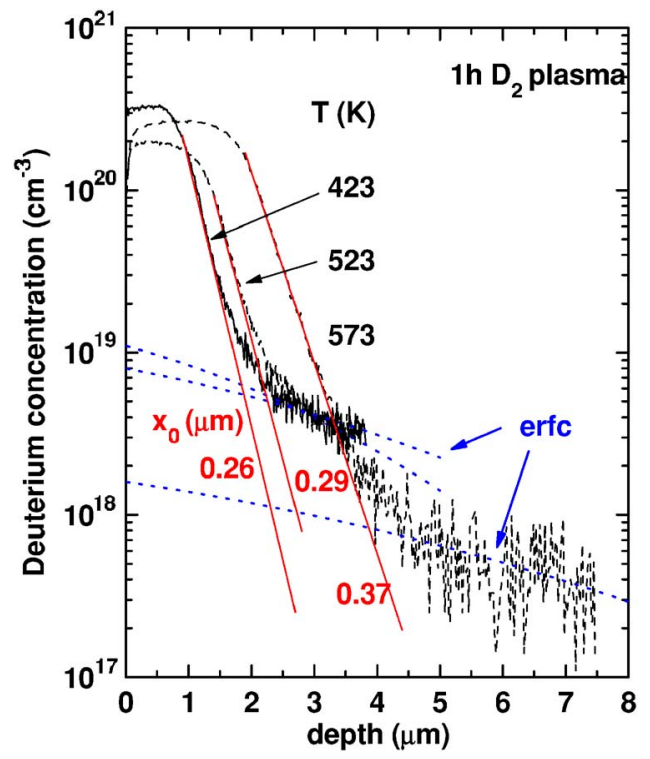

FIG. 3. (Color online) Deuterium concentration versus depth in polycrystalline $\mathrm{ZnO}$ obtained from SIMS measurements. The specimens were exposed to a deuterium plasma for $60 \mathrm{~min}$ at the indicated substrate temperatures. The dotted lines represent a leastsquares fit to Eq. (2), while the solid lines indicate an exponential decay of the $\mathrm{D}$ concentration.

hydrogen diffusion in disordered $\mathrm{ZnO}$ exhibits a fast and a slow diffusion component. While it is likely that fast diffusion occurs via interstitial lattice sites, the slow diffusion component is governed by a large concentration of deep trapping sites $\left(N_{\text {trap }}=2-3 \times 10^{20} \mathrm{~cm}^{-3}\right)$ that trap and release hydrogen on time scales long compared to the diffusion experiment.

The temperature dependence of the diffusion coefficient for low D concentration diffusion is plotted in Fig. 4. The diffusion coefficients are about one order of magnitude smaller than for D diffusion in single crystal $\mathrm{ZnO}$ (see Fig. 2). The data exhibit an apparent activation energy of $E_{A}$

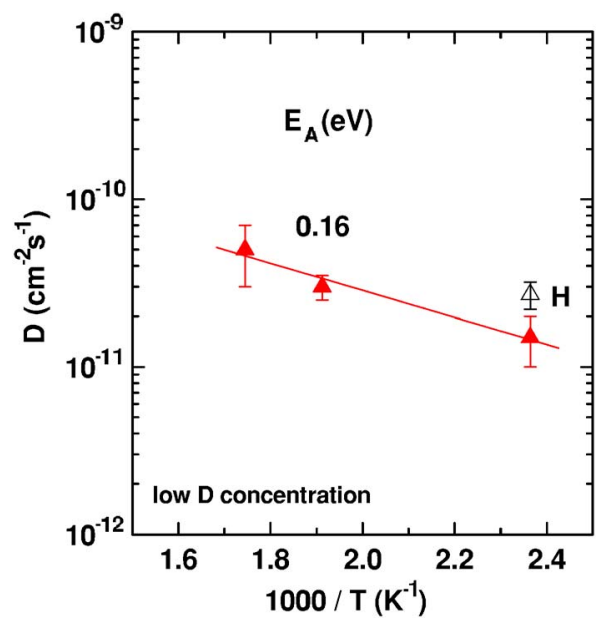

FIG. 4. (Color online) Diffusion coefficient in polycrystalline $\mathrm{ZnO}$ as a function of the reciprocal temperature for low D concentration diffusion. The full and open triangles represent deuterium and hydrogen diffusion experiments, respectively. 


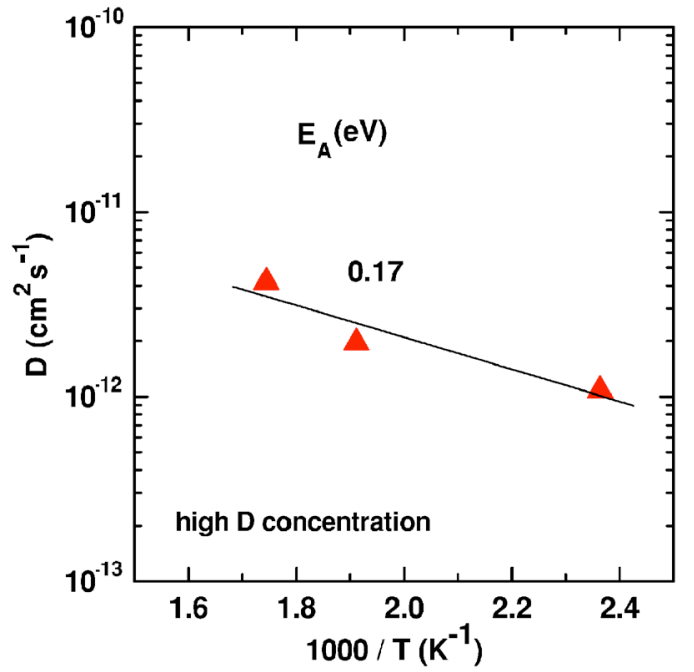

FIG. 5. (Color online) Temperature dependence of the diffusion coefficient for high $\mathrm{D}$ concentration diffusion in polycrystalline $\mathrm{ZnO}$.

$=0.16 \mathrm{eV}$ that is comparable to the data shown in Fig. 2. The low D concentration data (Fig. 3) exhibit some scattering. However, this does not cause a significant change of the apparent activation energy.

The $\mathrm{D}$ diffusion coefficients obtained for high deuterium diffusion (Fig. 5) are about one order of magnitude smaller than for low D concentration diffusion. Interestingly, the temperature dependence of $D$ exhibits a comparable activation energy of $E_{A}=0.16 \mathrm{eV}$. This is an unexpected result. Commonly, for diffusion in disordered semiconductors, one would expect a significant increase of the activation energy for low concentration diffusion, as was reported previously for polycrystalline silicon. ${ }^{14}$

\section{B. Hydrogen effusion}

Usually, hydrogen diffusion measurements rely on a process that initially introduces hydrogen or a tracer isotope, such as deuterium, into the sample. This step can be avoided by using hydrogen effusion measurements. Commonly, all semiconductors contain hydrogen atoms that were introduced during growth and/or subsequent processing steps. Hydrogen effusion measurements provide information on the total $\mathrm{H}$ concentration, the spectra of the $\mathrm{H}$ evolution rate as a function of temperature, the hydrogen density-of-states, and the diffusion coefficient. ${ }^{7,15,16}$

Figure 6 shows the molecular hydrogen flux of single crystal $\mathrm{ZnO}$ as a function of temperature. The four samples investigated were cut from one wafer. The effusion measurements were carried out with heating rates between 5 and $35 \mathrm{~K} / \mathrm{min}$. Significant out-diffusion of $\mathrm{H}_{2}$ occurs at temperatures above $470 \mathrm{~K}$. At temperatures above $1170 \mathrm{~K}$, the samples began to decompose resulting in an increase of the background pressure of the effusion setup. Hence, data taken at $T>1170 \mathrm{~K}$ cannot be analyzed reliably. It is interesting to note that the effusion spectra exhibit significant differences although all specimens were cut from one single crystal. This will be discussed in more detail in Sec. IV B. However, it is

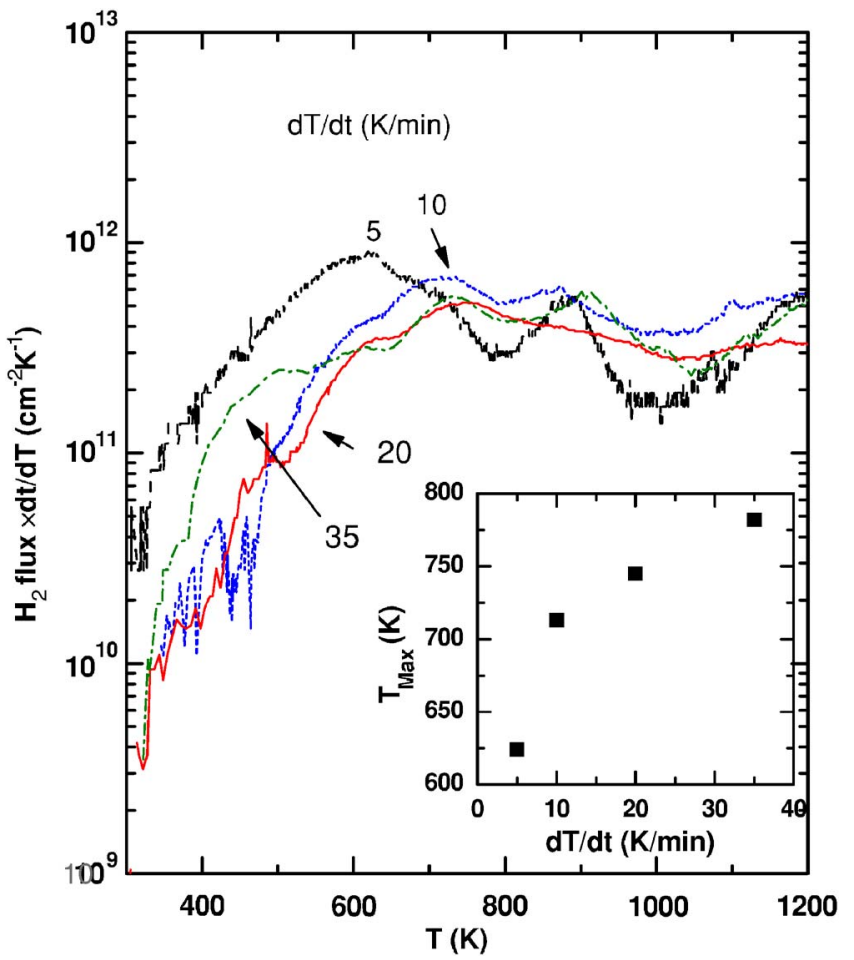

FIG. 6. (Color online) Molecular hydrogen evolution rate as a function of temperature. The measurements were performed on single crystal $\mathrm{ZnO}$ cut from one wafer. The measurements were performed with different heating rates varying between 5 and $35 \mathrm{~K} / \mathrm{min}$. The inset shows the temperature of the maximum $\mathrm{H}_{2}$ flux, $T_{\mathrm{Max}}$, as a function of the heating rate.

important to note that the maximum of the $\mathrm{H}_{2}$ flux shifts to larger temperatures as the heating rate is increased (see inset of Fig. 6). This is consistent with a previous report that showed that the hydrogen effusion mechanism in $\mathrm{ZnO}$ is governed by the diffusion of monatomic $\mathrm{H}$ from the bulk to the surface. ${ }^{7,17}$ Hence, the distribution of hydrogen in the sample, $C_{D}(x, t)$, has to be compatible with the diffusion equation

$$
\frac{\partial C}{\partial t}=D(t) \frac{\partial^{2} C}{\partial x^{2}},
$$

where the time-dependent diffusion coefficient is given by

$$
D(t)=D_{0} \exp \left(-\frac{E_{A}}{t k_{B} d T / d t}\right) .
$$

$D_{0}$ is the microscopic diffusion prefactor, $k_{B}$ is Boltzmann's constant, and $d T / d t$ is the heating rate. For a linear heating rate and an initially constant $\mathrm{H}$ concentration with depth, Beyer and Wagner ${ }^{15}$ derived the following equation to deduce the diffusion coefficient from hydrogen effusion data:

$$
\ln \left(\frac{d^{2}}{\pi^{2}} \frac{d T / d t}{k_{B} T_{\mathrm{Max}}^{2}}\right)=\ln \left(\frac{D_{0}}{E_{A}}\right)-\frac{E_{A}}{k_{B} T_{\mathrm{Max}}}=\ln \frac{D}{E_{A}} .
$$

Here, $T_{\mathrm{Max}}$ is the temperature of the maximum molecular hydrogen flux and $d$ is the sample thickness. Hence, the diffusion coefficient can be determined either from measuring 


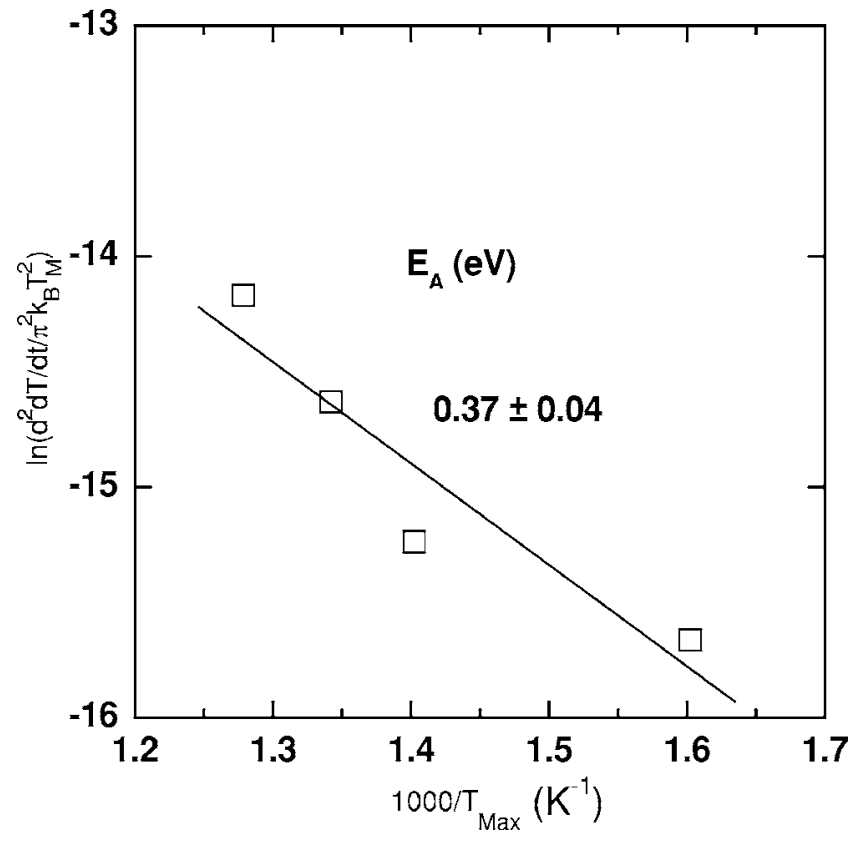

FIG. 7. $\ln \left[d^{2}(d T / d t) / \pi^{2} k_{B} T_{\text {Max }}^{2}\right]$ as a function of the reciprocal temperature $1 / T_{\mathrm{Max}}$.

$T_{\text {Max }}$ as a function of the sample thickness or as a function of the heating rate.

The analysis of the $\mathrm{H}$ effusion data with respect to the diffusion coefficient according to Eq. (5) is shown in Fig. 7. The solid line depicts a least-squares fit to the data and exhibits an activation energy of $E_{A}=0.37 \mathrm{eV}$. Compared to the values obtained for post-deuterated single crystal and polycrystalline $\mathrm{ZnO}$ the activation energy obtained from hydrogen effusion measurements is about a factor of 2 larger. From the data shown in Fig. 7, the diffusion coefficient

$$
D=D_{0} \exp \left(-E_{A} / k_{B} T\right)
$$

was determined and the values are plotted in Fig. 8 versus $1000 / T_{\text {Max }}$. These values have to be compared to the data shown in Fig. 2, which shows the diffusion coefficient for deuterium diffusion in single crystal $\mathrm{ZnO}$. While the effusion experiment yields data at higher temperatures they overlap at $1000 / T=1.6 \mathrm{~K}^{-1}$. At this temperature, the diffusion coefficient obtained from $\mathrm{H}$ effusion is about two orders of magnitude larger than $D$ obtained from SIMS measurements. Furthermore, when comparing the data of Fig. 8 to the diffusion coefficients obtained on polycrystalline $\mathrm{ZnO}$, there is also a difference in $D$ of about four orders of magnitude for both low and high deuterium concentration diffusion.

\section{DISCUSSION}

The experimental data presented in the previous section show that hydrogen diffusion in $\mathrm{ZnO}$ is a complex process. The data can be understood in terms of models that were developed to describe diffusion in disordered and single crystal silicon. Model parameters, such as the hydrogen chemical potential and hydrogen binding energies, can be extracted from the experimental data. This section is divided

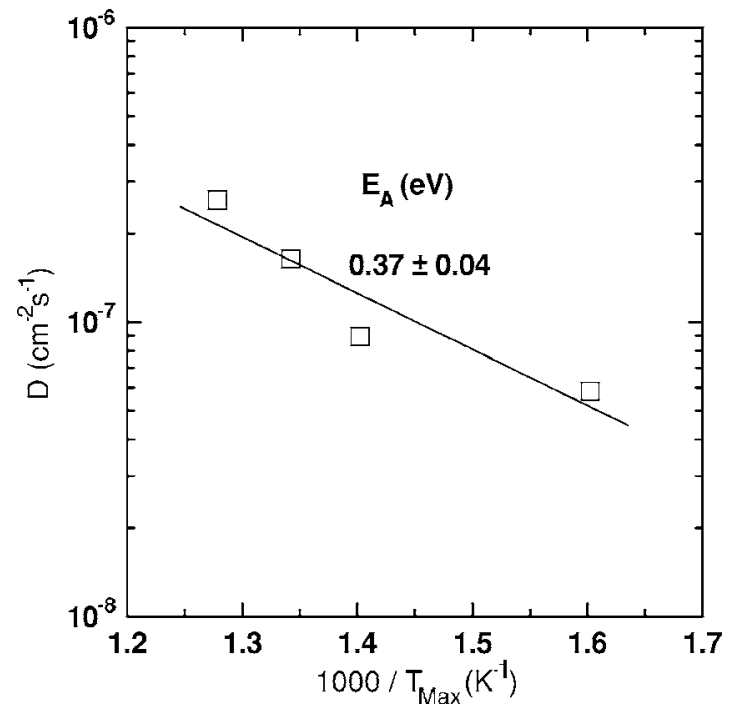

FIG. 8. Diffusion coefficient, $D$, as a function of $1 / T_{\text {Max }}$. The values for $D$ were obtained from the data shown in Fig. 7 .

into two parts. In the first part (Sec. IV A), the variation of the diffusion coefficient with temperature is examined. Then, in Sec. IV B, the role of the hydrogen density of states is discussed.

\section{A. Temperature dependence of the diffusion coefficient}

Already 50 years ago, hydrogen diffusion in $\mathrm{ZnO}$ has been studied intensively., 90 Thomas and Lander followed the migration of hydrogen by the change of the conductivity of the crystal upon exposure to hydrogen and found that $\mathrm{H}$ diffusion followed the relation

$$
2 D=0.063 \exp \left(-\frac{0.91 \mathrm{eV}}{k_{B} T}\right) .
$$

The origin of the factor of 2 arises from charge interaction from ionized $\mathrm{H}$ donors; the electrons are more mobile than the protons. Hence, they diffuse ahead in the space-charge region dragging the protons along. Mollwo 9 reported a somewhat larger activation energy of $E_{A}=1.12 \mathrm{eV}$.

Almost 50 years after the pioneering work of Mollwo and Thomas and Lander, a new investigation on hydrogen migration in $\mathrm{ZnO}$ claimed that the activation energy for $\mathrm{H}$ diffusion is as small as $E_{A}=0.17 \pm 0.12 \mathrm{eV}$, which would be consistent with the notion that atomic $\mathrm{H}$ diffuses in interstitial form. ${ }^{11}$ On first sight, our results presented above exhibit similarly small activation energies and seem to support the latter conclusion by Ip et al. ${ }^{11}$ However, when comparing diffusion coefficients and activation energies from different experiments (see Sec. III), it is obvious that the simple interpretation of the temperature dependence of $D$ as a measure for the migration barrier height is not valid.

Hydrogen transport occurs between minimum energy positions by surmounting the barrier between sites at a saddle point. Nominally undoped $\mathrm{ZnO}$ exhibits $n$-type conductivity. Recent theoretical and experimental research that focused on the origin of the $n$-type conductivity concluded that the pre- 


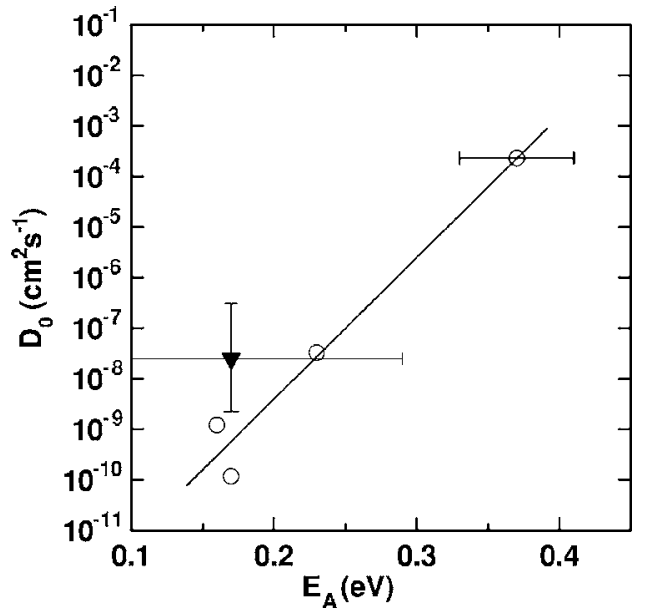

FIG. 9. Diffusion prefactor, $D_{0}$, as a function of the apparent activation energy $E_{A}$ determined from Figs. 2, 5, and 8 (open circles). The full triangle was determined from diffusion data of Ip et al. (Ref. 11).

ferred charge state of hydrogen is $\mathrm{H}^{+}{ }^{4-6,18}$ Hence, it is most likely that $\mathrm{H}$ migrates as a proton. In analogy to charge transport, it is commonly assumed that in a simple hydrogen density-of-states distribution the diffusion coefficient can be obtained from Eq. (6). In the past, it has been argued that this simple equation is valid for broad density of states that exhibit peaks at various energies. Moreover, based on these assumptions, $E_{A}$ should correspond to the energies of deep and shallow trapping sites. ${ }^{14,19}$ In the case of single crystal $\mathrm{ZnO}, E_{A}$ should reflect the barrier height between two transport sites. However, in this model, the key assumption is a diffusion prefactor, $D_{0}$, that is independent of all experimental parameters. From the experiments performed above the prefactor can be easily determined by extrapolating the temperature dependence of the diffusion coefficient to $1 / T$ $=0 \mathrm{~K}^{-1}$. Figure 9 shows the diffusion prefactor $D_{0}$ as a function of the apparent activation energy $E_{A}$. The open circles depict data from Sec. III that were obtained on single crystal and polycrystalline $\mathrm{ZnO}$. The full triangle was estimated from recent data published by Ip et al. ${ }^{11}$ They reported an activation energy of $E_{A}=0.17 \pm 0.12 \mathrm{eV}$ and a diffusion coefficient of $D \approx 8 \times 10^{-10} \mathrm{~cm}^{2} \mathrm{~s}^{-1}$ at $573 \mathrm{~K}$. With increasing activation energy, the diffusion prefactor increases by about eight orders of magnitude. The data can be described by a straight line independent of the measurement method and the degree of disorder. Such a relation is well know as MeyerNeldel behavior. ${ }^{20}$ Previously, it was also observed for hydrogen diffusion in amorphous, microcrystalline, and polycrystalline silicon. ${ }^{21,22}$ Figure 9 shows that the prefactor $D_{0}$ depends on the experimental conditions. Therefore, the apparent activation energies, $E_{A}$, derived from the temperature dependence of the diffusion coefficient are not related to the energetic position of shallow trapping sites or the barrier height between the transport sites.

The microscopic diffusion prefactor should be constant and independent of experimental conditions such as degree of disorder, $\mathrm{H}$ concentration, passivation temperature, and experimental methods. From a theoretical approach, the microscopic diffusion prefactor is given by

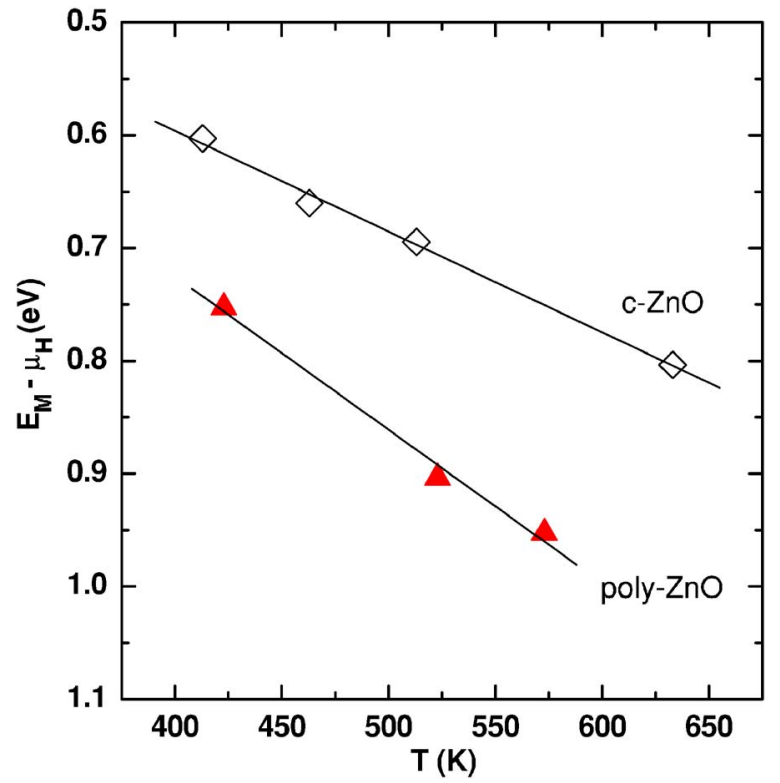

FIG. 10. (Color online) Position of the hydrogen chemical potential, $E_{M}-\mu_{H}(T)$, as a function of temperature. The open diamonds and full triangles represent deuterium diffusion in single crystal and polycrystalline $\mathrm{ZnO}$, respectively.

$$
D_{0}^{T}=\frac{1}{N_{H}} \nu a^{2},
$$

where $N_{H}$ is the number of nearest-neighbors sites, $\nu$ is the attempt frequency, and $a$ is the mean-free path. Depending on the preferred migration site for $\mathrm{H}^{+}, N_{H}$ is either 3 for a migration path via hydrogen at an oxygen antibonding site or 6 for a migration path via the oxygen bonding sites. Using reasonable values of $\nu=10^{13} \mathrm{~Hz}$ and $a \approx 4 \AA$, the diffusion prefactor can be estimated to $D_{0}^{T} \approx 0.7-5 \times 10^{-3} \mathrm{~cm}^{2} \mathrm{~s}^{-1}$. This is in good agreement with the experimentally obtained diffusion prefactor of $D_{0}=3 \times 10^{-2} \mathrm{~cm}^{2} \mathrm{~s}^{-1}$ from Thomas and Lander. ${ }^{10}$ Using the microscopic diffusion prefactor, the position of the hydrogen chemical potential, $\mu_{H}(T)$, can be estimated with respect to the migration saddle point, $E_{M}$, using the relation

$$
E_{M}-\mu_{H}(T)=-k_{B} T \ln \left(\frac{D}{D_{0}^{T}}\right) .
$$

For this purpose, a value of $D_{0}^{T} \simeq 10^{-3} \mathrm{~cm}^{2} \mathrm{~s}^{-1}$ was used. This does not produce a large error of the following analysis since the results depend only logarithmically on the assumed value of the microscopic diffusion prefactor; an error of one order of magnitude will cause an energy change of less than $100 \mathrm{meV}$.

The temperature dependence of the position of the hydrogen chemical potential is shown in Fig. 10 for diffusion in single crystal (open diamonds) and polycrystalline $\mathrm{ZnO}$ (full triangles). With increasing temperature, the chemical potential sinks deeper in energy with respect to $E_{M}$ at roughly a rate of $0.0013 \pm 0.0008 \mathrm{eV} / \mathrm{K}$. This rapid change of $\mu_{H}$ is only possible if the concentration of $\mathrm{H}$ trapping sites is small at the chemical potential. The temperature dependence of $\mu_{H}$ 
is most likely related to the hydrogen quasichemical potential of the $\mathrm{H}$ plasma. Assuming that $\mathrm{H}$ in the $\mathrm{ZnO}$ solid is in equilibrium with the monatomic $\mathrm{H}$ in the plasma, then a change of the plasma quasichemical potential, $\mu_{P}$, will cause a change of $E_{M}-\mu_{H}$. In the plasma, $\mu_{P}$ is given by ${ }^{23}$

$$
\mu_{P}=k_{B} T \ln \left(c V_{Q}\right),
$$

where $T$ is the gas temperature, $c$ is the atomic $\mathrm{H}$ concentration, and $V_{Q}$ is the quantum volume. According to Ref. 13 $c$ amounts to about $5 \times 10^{15} \mathrm{~cm}^{-3}$. At $300 \mathrm{~K}$, the quantum volume is equal to $V_{Q}=10^{-24} \mathrm{~cm}^{3} .{ }^{23}$ With these values, the temperature dependence of the plasma quasichemical potential can be calculated to about $\mu_{P} / T=-0.0016 \mathrm{eV} / \mathrm{K}$. This change is in good agreement with the values of $\approx-0.0013 \pm 0.0008 \mathrm{eV} / \mathrm{K}$ obtained from the data in Fig. 10 . The decrease of the chemical potential occurs because with increasing temperature hydrogen in the solid state is less favorable than $\mathrm{H}$ in the gaseous state due to the greater entropy.

Using the temperature dependence of the hydrogen chemical potential, the data can be corrected to calculate $E_{M}-\mu_{H}(T)$ at a constant temperature. At a temperature of $350{ }^{\circ} \mathrm{C}$, the hydrogen chemical potential resides at 0.79 and $1.02 \mathrm{eV}$ below the migration saddle point for single crystal and polycrystalline $\mathrm{ZnO}$, respectively. These values for the $\mathrm{H}$ chemical potential are compatible with the diffusion activation energy of 0.91 and 1.12 by Thomas and Landner ${ }^{10}$ and Mollwo. ${ }^{9}$

The temperature dependence of the diffusion coefficient obtained from hydrogen effusion measurements cannot be analyzed by the method presented above. However, from the molecular hydrogen flux, the position of the hydrogen chemical potential can easily be derived using the relation ${ }^{16}$

$$
E_{M}-\mu_{H}=-k_{B} T \ln \left(\frac{F_{H_{2}}}{F_{0}}\right)
$$

where the prefactor is given by $F_{0} \approx 2 \nu a N_{\text {Surf }} / d$. Here, $d$ is the sample thickness and $N_{\text {Surf }} \approx 10^{15} \mathrm{~cm}^{-2}$ is the number of $\mathrm{H}$ surface states. With $d \times F_{0} \approx 6 \times 10^{20} \mathrm{~cm}^{-1} \mathrm{~s}^{-1}$, the position of the $\mathrm{H}$ chemical potential can be calculated. In Fig. 11, $E_{M}-\mu_{H}$ is depicted as a function of the hydrogen concentration for single crystal $\mathrm{ZnO}$ measured with heating rates of 5, 20 , and $35 \mathrm{~K} / \mathrm{min}$. At an $\mathrm{H}$ concentration approaching $10^{18} \mathrm{~cm}^{-3}$, the $\mathrm{H}$ chemical potential resides about $0.6 \mathrm{eV}$ below $E_{M}$. Independent of the heating rate, $E_{M}-\mu_{H}$ increases rapidly to a value between 2.0 and $2.3 \mathrm{eV}$ as the residual hydrogen concentration in the sample approaches a value of $10^{17} \mathrm{~cm}^{-3}$. As the residual $\mathrm{H}$ concentration decreases further, the $\mathrm{H}$ chemical potential remains constant. This is indicative of a negative-U system. ${ }^{24}$

\section{B. Hydrogen density of states}

The analysis of the presented data has shown that with increasing temperature (Fig. 10) and decreasing hydrogen content (Fig. 11), the $\mathrm{H}$ chemical potential shifts readily toward deeper energies with respect to the transport sites, independent of the method used to investigate hydrogen diffu-

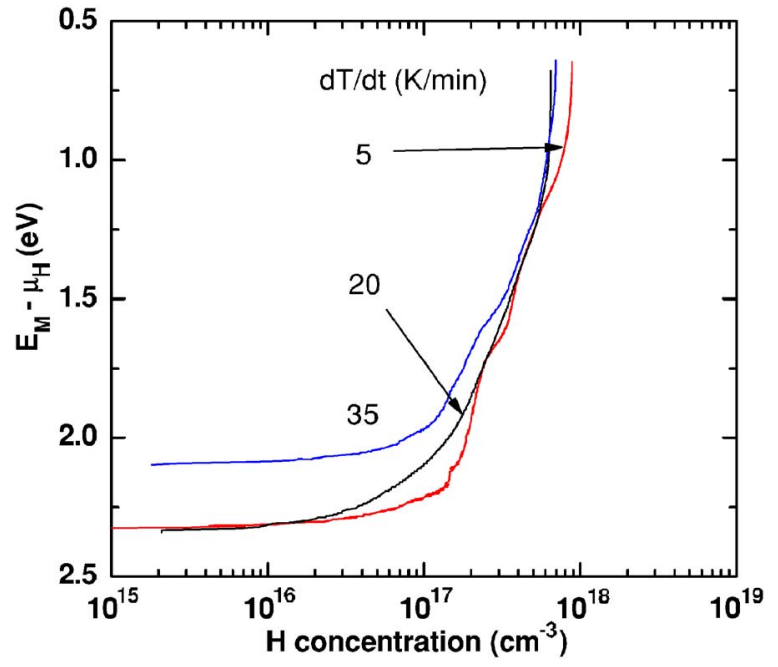

FIG. 11. (Color online) Hydrogen chemical potential with respect to the migration saddle point, $E_{M}-\mu_{H}$, as a function of the hydrogen concentration. The data were derived from the effusion spectra shown in Fig. 6. For clarity, the data for a heating rate of $10 \mathrm{~K} / \mathrm{min}$ are omitted.

sion in $\mathrm{ZnO}$. The observed behavior suggests that the hydrogen chemical potential resides above a minimum in the density of states. The deuterium concentration depth profiles (Figs. 1 and 3) contain some more information regarding the $\mathrm{H}$ density of states. The exponential depth dependence of the high D concentration part of the depth profiles indicates that $\mathrm{H}$ is being trapped at energetically deep sites. Their concentration amounts to $N_{\text {trap }}=0.4-3 \times 10^{20} \mathrm{~cm}^{-3}$ (see Figs. 1-3). From the present set of data, information on the energetic position cannot be obtained. However, since the D concentration exhibits an exponential depth dependence, one can conclude that these sites reside significantly deeper in energy than the $\mathrm{H}$ transport sites, since an exponential decay is indicative of sites that trap and release hydrogen on timescales much longer than those of the diffusion experiments.

If hydrogen were simply to diffuse through the $\mathrm{ZnO}$ samples, one would not expect to observe the buildup of large $\mathrm{H}$ concentrations in the near-surface region. This observation (Figs. 1 and 3) demonstrates that hydrogen has a tendency to create additional trapping sites. This is a wellknown phenomenon in other semiconductors. In $n$-type single crystal and polycrystalline silicon, high deuterium concentration depth profiles indicate the presence of platelets. These structural defects are believed to be a consequence of the coordination formation of $\mathrm{Si}-\mathrm{H}$ bonds driven by strain. ${ }^{25-27}$ Other possibilities include, but are not limited to, the neutralization of dopants and impurities, passivation of structural defects, such as interstitials, vacancies, and stacking faults. Although interesting and important, the identification of the microscopic origin of the near-surface D accumulation is beyond the scope of this research and requires a significant amount of additional work.

Information on the nature of the $\mathrm{H}$ density of states can be derived from the $\mathrm{H}$ effusion spectra. The total $\mathrm{H}$ concentration of any semiconductor is given by 


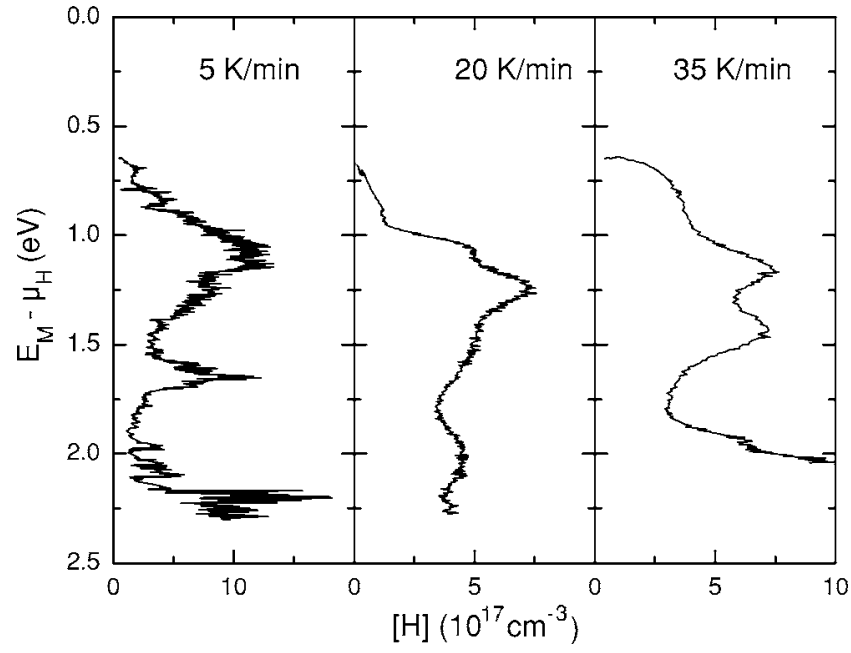

FIG. 12. Hydrogen density of states distribution of single crystal $\mathrm{ZnO}$ cut from one wafer. The data were obtained by applying Eq. (13) to the hydrogen effusion data shown in Fig. 6.

$$
C_{H}=\int N_{H}(E) f\left(T, E, \mu_{H}\right) d E .
$$

In this equation, $N_{H}$ denotes the hydrogen density of states, and $f\left(T, E, \mu_{H}\right)$ represents the occupation function. Since the occupation function $f$ peaks at $E=\mu_{H}$ the $\mathrm{H}$ density of states is given by ${ }^{16}$

$$
N_{H}\left(\mu_{H}\right) \approx \frac{\partial C_{H}}{\partial \mu_{H}} .
$$

In Fig. 12, the hydrogen DOS is shown for 3 single crystal $\mathrm{ZnO}$ samples. A significant concentration of $\mathrm{H}$ sites occurs for $\mathrm{H}$ binding energies between 1.0 and $1.3 \mathrm{eV}$. This is consistent with previously published data. ${ }^{7}$ However, it is interesting to note that the $\mathrm{H}$ density of states differs significantly for the investigated samples suggesting that this is due to a change of the heating rate. Commonly, however, this is not the case. When changing the heating rate or the sample thickness, one usually obtains the same $\mathrm{H}$ density of states. This has been demonstrated experimentally for sputter deposited $\mathrm{ZnO}$ samples. ${ }^{7}$ Hence, the data shown in Fig. 12 indicate that in single crystal $\mathrm{ZnO}$, the $\mathrm{H}$ distribution and the $\mathrm{H}$ density of states vary significantly over the area of a wafer. According to the $\mathrm{H}$ density of states, most $\mathrm{H}$ atoms are accommodated at binding energies between $E_{M}-\mu_{H}=1$ and
$1.5 \mathrm{eV}$. No data were obtained for binding energies of less than $E_{M}-\mu_{H}=0.8 \mathrm{eV}$ clearly demonstrating that a diffusion activation energy of less than $0.8 \mathrm{eV}$ is highly unlikely. Therefore, the measured $\mathrm{H}$ density of states is consistent with the original $\mathrm{H}$ diffusion measurements of Mollwo ${ }^{9}$ and Thomas and Lander. ${ }^{10}$

\section{SUMMARY}

In summary, we have investigated hydrogen diffusion in single crystal and polycrystalline $\mathrm{ZnO}$ by using posthydrogenation experiments followed by SIMS measurements and hydrogen effusion measurements. The deuterium depth profiles exhibited a fast and a slow diffusion component in both sets of $\mathrm{ZnO}$ samples with an apparent activation energy of $E_{A}=0.17 \mathrm{eV}$. Only the temperature dependent diffusion coefficient, deduced from $\mathrm{H}$ effusion experiments, exhibited a larger apparent activation energy of $E_{A}=0.34 \mathrm{eV}$. Although these values are consistent with a recently reported activation energy by Ip et al. ${ }^{11}$ they are about one order of magnitude smaller than $E_{A}$ from the original works of Mollwo ${ }^{9}$ and Thomas and Lander. ${ }^{10}$ However, the variation of the observed apparent activation energy is accompanied by a change of the diffusion prefactor $D_{0}$ by about eight orders of magnitude. This shows that $E_{A}$ does not represent the barrier height for $\mathrm{H}$ migration. Using the theoretical diffusion prefactor, the position of the $\mathrm{H}$ chemical potential was calculated as a function of temperature. $E_{M}-\mu_{H}$ increases with a rate of about $0.001 \mathrm{eV} / \mathrm{K}$ that is due to a decrease of the plasma chemical potential. In response to a decrease of the $\mathrm{H}$ concentration from $10^{18}$ to $10^{17} \mathrm{~cm}^{-3} \mu_{H}$ shifts about $2.5 \mathrm{eV}$ away from the $\mathrm{H}$ transport states. As the residual $\mathrm{H}$ concentration decreases further, the $\mathrm{H}$ chemical potential is pinned which is indicative of a negative $\mathrm{U}$ system. The $\mathrm{H}$ density of states distribution derived from $\mathrm{H}$ effusion data shows that most $\mathrm{H}$ atoms are accommodated with binding energies of 1.0 to $1.5 \mathrm{eV}$. The lack of trapping sites with binding energies of less than about $0.8 \mathrm{eV}$ is consistent with the original $\mathrm{H}$ diffusion measurements of Mollwo ${ }^{9}$ and Thomas and Lander. $^{10}$

\section{ACKNOWLEDGMENTS}

The author wishes to thank D. Kosarev of the Materials Analysis Group in Sunnyvale, CA, who carried out the SIMS measurements. Technical support is acknowledged from J. Krause and D. Patzek.
${ }^{1}$ Zinc Oxide-A Material for Micro- and Optoelectronic Applications, NATO Science Series II Vol. 194, edited by N. H. Nickel and E. Terukov (Springer, New York 2005).

${ }^{2}$ J. F. Wager, Science 300, 1245 (2003).

${ }^{3}$ D. G. Thomas, J. Phys. Chem. Solids 15, 86 (1960).

${ }^{4}$ C. G. Van de Walle, Phys. Rev. Lett. 85, 1012 (2000).

${ }^{5}$ S. F. J. Cox, E. A. Davis, S. P. Cottrell, P. J. C. King, J. S. Lord, J. M. Gil, H. V. Alberto, R. C. Vilão, J. Pironto Duarte, N. Ayres de Campos, A. Weidinger, R. L. Lichti, and S. J. C. Irvine, Phys. Rev. Lett. 86, 2601 (2001).

${ }^{6}$ D. M. Hofmann, A. Hofstaetter, F. Leiter, H. Zhou, F. Henecker, B. K. Meyer, S. B. Orlinskii, J. Schmidt, and P. G. Baranov, Phys. Rev. Lett. 88, 045504 (2002).

${ }^{7}$ N. H. Nickel and K. Brendel, Phys. Rev. B 68, 193303 (2003).

${ }^{8}$ N. H. Nickel and K. Fleischer, Phys. Rev. Lett. 90, 197402 (2003). 
${ }^{9}$ E. Mollwo, Z. Phys. 138, 478 (1954).

${ }^{10}$ D. G. Thomas and J. J. Lander, J. Chem. Phys. 25, 1136 (1956).

${ }^{11}$ K. Ip, M. E. Overberg, Y. W. Heo, D. P. Nortgon, S. J. Pearton, C. E. Stutz, B. Luo, F. Ren, D. C. Look, and J. M. Zavada, Appl. Phys. Lett. 82, 385 (2003).

${ }^{12}$ Single-crystal $\mathrm{ZnO}$ samples were obtained from Eagle-Picher and Cermet.

${ }^{13}$ N. M. Johnson, J. Walker, and K. W. Stevens, J. Appl. Phys. 69, 2631 (1991).

${ }^{14}$ N. H. Nickel, W. B. Jackson, and J. Walker, Phys. Rev. B 53, 7750 (1996).

${ }^{15}$ W. Beyer and H. Wagner, J. Appl. Phys. 53, 8745 (1982).

${ }^{16}$ W. B. Jackson, A. J. Franz, H.-C. Jin, J. R. Abelson, and J. L. Gland, J. Non-Cryst. Solids 227, 143 (1998).

${ }^{17} \mathrm{~W}$. Beyer, in Hydrogen in Semiconductors II, edited by N. H. Nickel (Academic, San Diego, 1999), Vol. 61, p. 165.

${ }^{18}$ D. C. Look, D. C. Reynolds, J. R. Sizelove, R. L. Jones, C. W. Litton, G. Cantwell, and W. C. Harsch, Solid State Commun.
105, 399 (1998).

${ }^{19}$ P. V. Santos and W. B. Jackson, Phys. Rev. B 46, 4595 (1992).

${ }^{20}$ W. Meyer and H. Neldel, Z. Tech. Phys. (Leipzig) 18, 588 (1937).

${ }^{21}$ N. H. Nickel and I. E. Beckers, Phys. Rev. B 66, 075211 (2002).

${ }^{22}$ W. Beyer, Phys. Status Solidi A 159, 53 (1997).

${ }^{23}$ C. Kittel, Thermal Physics (Wiley, New York, 1969), p. 164.

${ }^{24}$ The expression "negative-U" system comes from the correlation energy $U$ which is a consequence of the Coulomb repulsion of electrons at one site. In a negative-U system, the acceptor state resides above the donor state.

${ }^{25}$ N. M. Johnson, F. A. Ponce, R. A. Street, and R. J. Nemanich, Phys. Rev. B 35, 4166 (1987).

${ }^{26}$ N. H. Nickel, G. B. Anderson, and J. Walker, Solid State Commun. 99, 427 (1996).

${ }^{27}$ N. H. Nickel, G. B. Anderson, N. M. Johnson, and J. Walker, Phys. Rev. B 62, 8012 (2000). 